\title{
Gegen „schwitzige“ Hände und Füße
}

\author{
Der Kosmetikhersteller Sweat-off, \\ spezialisiert auf Antitranspirante, \\ bietet seit kurzem ein neues Pro- \\ dukt namens "Sweat-off hands \\ and feet" an. Es wurde speziell für \\ die Hautstruktur an Händen und \\ Füßen entwickelt und reduziert \\ das Schwitzen an den Handinnen- \\ flächen und Fußsohlen auf ein na- \\ türliches Maß. Bei regelmäßgier \\ Anwendung sorgt es tagelang für \\ ein trockenes und griffiges Haut- \\ gefühl.
}

Das neue Antitranspirant muss zu Beginn abends aufgetragen werden und zieht schnell in die Hautstruktur ein. Nach drei bis vier Wochen minimiert es das Schwitzen auf ein natürliches Maß.

Für die Wirksamkeit und Verträglichkeit sorgen als Inhaltsstoffe unter anderem Aluminiumsalze und pflanzliche Zellulose. Die Antitranspirante von Sweat-off enthalten keinerlei Alkohol, Parfüm und Konservierungsmittel. Nach dem Auftragen lösen sich die Inhaltsstof- fe des Antitranspirants auf der warmen Haut. Die enthaltenen Aluminiumsalze bilden einen Film, der sich über die Poren legt und die Schweißdrüsen an ihrer Oberseite verengt. Auf diese Weise wird an den behandelten Hautflächen die Schweißbildung deutlich reduziert. Uneffektiver Flüssigkeitsverlust ohne Temperaturregelung des Körpers wird vermieden, der Körper kann wieder an Regionen schwitzen, die vermehrt Kühlungseffekt bringen.

Anders als Deos müssen Antitranspirante nicht täglich oder mehrmals am Tag aufgetragen werden, sondern nach einer ersten „Eingewöhnungsphase“ nur noch wenn es nötig ist. Die ersten Male genügt es, an maximal drei bis vier Abenden vor dem Schlafengehen das Mittel auf die frisch gewaschene, trockene Haut aufzutragen. Anschließend reicht die wiederholte Anwendung, wenn die Wirkung des Antitranspirants nachlässt. Das kann nach einigen Tagen oder wenigen Wochen sein - je nach Witterung und persönlichen Umständen.

\section{Perfekt kombinierte Reinigung und Pflege für irritierte Haut}

\author{
Irritierte, barrieregeschädigte \\ Haut benötigt besonders intensi- \\ ve und vitalisierende Pflege. Op- \\ timal aufeinander abgestimmte \\ Präparate sind dabei das A und \\ $O$ der Hautpflege. Deshalb bietet \\ Dermasence jetzt zusätzlich zur \\ etablierten, barriereaufbauenden \\ BarrioPro Gesichtsemulsion zwei \\ neue Ergänzungen zur Reini- \\ gung und Pflege der barriere- \\ geschädigten Haut.
}

Zur sanften Reinigung für Gesicht und Körper wurde der neue anti-irritative Dermasence BarrioPro Reinigungsschaum entwickelt. Er reinigt und beruhigt sensible Haut, die zu Rötungen und Juckreiz neigt. Färberwaidextrakt wirkt antiinflammato- risch und antimykotisch, bringt die Mikroflora ins Gleichgewicht und optimiert so die Widerstandskraft der Haut. Glycerin bewirkt einen hydratisierenden Effekt. Der ergiebige Schaum ermöglicht eine wasserfreie Reinigung und eignet sich auch bei perioraler Dermatitis, bei diabetischen Hautproblemen und zur Rasur empfindlicher Haut.

Zur anschließenden hauttypgerechten Pflege des Körpers ist die neue Dermasence BarrioPro Körperemulsion, eine feuchtigkeitsspendende Körperpflege mit Sanddornfruchtfleischöl, eine ideale Ergänzung. Durch die Synergie aus Pflanzenextrakten, einem Polysaccharid, Vitamin A und Palmitoylethanolamid („PEA“) wird ein antiirritativer Effekt erzielt. Der Inhaltsstoff PEA enthält Palmitinsäure, die einen natürlichen Bestandteil der
Hautbarriere darstellt. Ein Hautrezeptor wird stimuliert und Entzündungsvorgänge werden eingedämmt. Derselbe Rezeptor ist für die Hautreifung und Versorgung der Epidermis mit Barrierelipiden zuständig, so dass die Körperemulsion den physiologischen Hautaufbau effektiv unterstützt. Bioaktive Substanzen aus extrahiertem Sanddornfruchtfleisch wie Vitamin E und C stärken das antioxidative Schutzsystem der Haut und tragen zur antiinflammatorischen Wirkung bei. Vitamin A stärkt die Hautbarriere und übernimmt regulatorische Funktionen der Reparaturmechanismen der geschädigten Haut. Die angenehme Galenik zieht schnell ein und verleiht der Haut Geschmeidigkeit, ohne einen Fettfilm zu hinterlassen.

Das „BarrioPro-Programm“ für irritierte Haut ist ab sofort in allen Apotheken erhältlich. Weitere Informationen und Proben können kostenlos beim Außendienst oder telefonisch unter 02504/9332-300 angefordert werden.

Nach Informationen von P\&M Cosmetics 